\title{
Influencia de la concentración de pigmento en volumen (CPV) en las propiedades de una imprimación epoxi
}

\author{
M.T. Rodríguez*, S.J. García*, J.J. Gracenea**, K.A. Habib* y J.J. Suay* \\ Resumen Mediante ensayos de tracción (tensión-deformación), ensayos electroquímicos (EIS y \\ AC/DC/AC) y ensayos acelerados de niebla salina, se ha estudiado el efecto del parámetro \\ $\Lambda=\mathrm{CPV} / \mathrm{CCPV}$ (siendo CCPV la concentración de pigmento en volumen crítica) en las \\ propiedades mecánicas y anticorrosivas de una imprimación epoxi. Se formularon \\ imprimaciones con diferentes valores de $\Lambda(0,73,0,80,0,88,0,93,0,98,1,08,1,15$ y 1,24$)$. \\ Se ha observado que las propiedades mecánicas y anticorrosivas de una imprimación epoxi \\ se ven altamente afectadas por el parámetro $\Lambda$, aumentando la resistencia a corrosión al \\ disminuir la CPV. Las conclusiones halladas mediante las diferentes técnicas fueron muy \\ similares, destacando los ensayos AC/DC/AC por su rapidez en la obtención de resultados \\ (24 h).
}

Palabras clave CPV. Epoxi. EIS. AC/DC/AC. Imprimación.

\section{Influence of the pigment volume concentration (PVC) on the properties of an epoxy primer}

\begin{abstract}
By means of stress-strain tests, electrochemical techniques (EIS and AC/DC/AC) and salt spray accelerated tests, the influence of the $\Lambda=$ PVC/CPVC parameter (being CPVC the critical pigment volume concentration) on mechanical and anticorrosive properties of an anticorrosive epoxy organic primer has been studied. Primers were formulated at different $\Lambda$ values $(0.73,0.80,0.88,0.93,0.98,1.08,1.15$ and 1.24$)$. It was concluded that mechanical and anticorrosive properties of an epoxy coating are greatly affected by the $\Lambda$ parameter. The corrosion resistance increases when the PVC decreases. Anticorrosive properties characterized with the different techniques used were very similar, although $\mathrm{AC} / \mathrm{DC} / \mathrm{AC}$ offered the results in a very short time $(24 \mathrm{~h})$.
\end{abstract}

Keywords

PVC. Epoxy. EIS. AC/DC/AC. Primer.

\section{INTRODUCCIÓN}

La concentración de pigmento en volumen crítica (CCPV) fue definida por primera vez, en 1949, por Asbeck y Van Loo ${ }^{[1]}$. Diferentes estudios han demostrado que la adición de pigmento tiene una fuerte influencia en las propiedades de la imprimación y de forma particular cuando CPV $=\mathrm{CCPV}$ $(\wedge=1)$, se produce un drástico cambio en las características del recubrimiento ${ }^{[2]}$. Para valores de CPV menores que CCPV, la imprimación seca se comporta como una matriz polimérica continua con los pigmentos distribuidos al azar, en su seno. Si CPV es mayor que CCPV no hay suficiente resina como para poder recubrir todas las partículas de pigmen- to, por lo que se crean estructuras con huecos ocupados por aire. Así, la nueva fase presente (aire) afecta de manera considerable a las propiedades del recubrimiento, especialmente a la densidad, propiedades mecánicas, térmicas, de transporte, anticorrosivas y ópticas ${ }^{[3]}$.

Actualmente, el efecto del ratio CPV/CCPV sobre las propiedades anticorrosivas se estudia por medio de diferentes ensayos como el de resistencia a niebla salina ${ }^{[4]}$. Sin embargo, debido a los inconvenientes propios de estas técnicas (no ofrecen información de los procesos de corrosión que tienen lugar en la interfase del metal), se han buscado alternativas basadas en estudios electroquímicos, como es el uso de la espectroscopia de impedancia

(*) Dpto. de Tecnología, Área de Ciencia de los Materiales e Ingeniería Metalúrgica, Universitat Jaume I, Av. de Vicent Sos Baynat s/n, 12071 Castellón, España.

(**) Naber Pinturas y Barnices, S.L. N-332, Km 279, 46469 Beniparrell, Valencia, España. 
electroquímica (EIS, en siglas inglesas) ${ }^{[3 \text { y } 5-10]}$. No obstante, ambas técnicas necesitan exponer las muestras a la presencia de electrolito durante largos periodos de tiempo, para producir su degradación. Esto ha propiciado la búsqueda de nuevos ensayos de evaluación que requieran tiempos de exposición inferiores. Hollaender et.al. ${ }^{[11-13]}$ desarrollaron un método de evaluación acelerado, consistente en someter la muestra a polarizaciones catódicas y ensayos EIS, alternativamente. Esta técnica, atribuye los espectros de impedancia obtenidos en los ensayos EIS a los procesos de degradación de la imprimación (aparición de poros y deslaminación del recubrimiento), provocados por la formación de $\mathrm{H}_{2}$ y $\mathrm{OH}^{-}$en la interfase metal-imprimación como resultado de la polarización catódica.

El presente trabajo se centra en el estudio del efecto de la variación del parámetro $\Lambda$ en las propiedades de una imprimación epoxi, en la presentación de la técnica AC/DC/AC como método eficaz y rápido para caracterizar las propiedades anticorrosivas, y en la comparación de los resultados obtenidos mediante esta técnica con el ensayo electroquímico EIS y el de resistencia a niebla salina.

\section{MATERIALES Y PROCEDIMIENTO EXPERIMEN- TAL}

\subsection{Pinturas}

Las imprimaciones empleadas en el ensayo se formularon usando una resina epoxi basada en diglicil eter de bisfenol $\mathrm{A}$, un agente de curado basado en una poliamina alifática y una amina terciaria como catalizador. Los valores de $\Lambda$ se calcularon mediante la ecuación (1) experimental, referenciada por Hare y Kurnas ${ }^{[14]}$ :

$$
\mathrm{CCPV}=1 /\left(1+\sum_{i=1}^{P} X_{i} \cdot d_{i} \cdot \AA_{i} / K\right)
$$

donde, $i$ indica los diferentes tipos de pigmento presentes $(i=1,2,3 \ldots \mathrm{P}), X_{i}$ es la fracción volumétrica de cada pigmento, $\delta_{\mathrm{i}}$ es la gravedad específica de cada pigmento y $\widetilde{A}_{i}$ es el valor de la absorción de aglutinante de cada pigmento. Como el aceite de linaza es el aglutinante universalmente utilizado en este ensayo (norma ASTM D 281) el valor de K empleado es 93,5 , y los datos de $\widetilde{A}_{i}$ están referidos a este aceite. Algunas de las características de las imprimaciones formuladas se pueden ver en la tabla I.
Tabla I. Características de las imprimaciones estudiadas

Table I. Studied primers characteristics

\begin{tabular}{cccc}
\hline Sol. en Peso (\%) & Sol. en Volumen & CPV (\%) & CPV/CCPV \\
\hline 70,8 & 55,4 & 36,00 & 0,73 \\
70,3 & 53,7 & 39,66 & 0,80 \\
69,8 & 52,2 & 43,28 & 0,88 \\
69,5 & 51,1 & 45,82 & 0,93 \\
69,2 & 50,0 & 48,62 & 0,98 \\
68,7 & 48,3 & 53,19 & 1,08 \\
68,4 & 47,0 & 57,00 & 1,15 \\
68,0 & 45,5 & 61,38 & 1,24 \\
\hline
\end{tabular}

\subsection{Equipos y métodos de ensayo}

\subsubsection{Ensayo de tracción}

Los ensayos se realizaron con una máquina universal de ensayos INSTRON empleando una célula de carga de $100 \mathrm{~N}$. Las muestras fueron curadas a temperatura ambiente durante una semana. Los ensayos de tracción siguieron la norma ASTM D1708-96 ${ }^{[4]}$.

\subsubsection{Espectroscopia de Impedancia Electroquími- ca (EIS)}

Se realizaron ensayos EIS sobre muestras recubiertas con las imprimaciones epoxis formuladas. Como electrolito se utilizó una disolución de agua destilada al 3,5\% de $\mathrm{NaCl}$ en peso. La celda electroquímica con tres electrodos se estableció fijando un cilindro de vidrio sobre la superficie del sustrato, llenándolo con el electrolito mencionado y utilizando como contraelectrodo una lámina de grafito, como electrodo de referencia uno de $\mathrm{Ag} / \mathrm{AgCl}$ saturado y como electrodo de trabajo el mismo sustrato metálico. El área expuesta al electrolito fue de $16,6 \mathrm{~cm}^{2}$.

El ensayo de impedancia fue realizado con un potenciostato y analizador de la respuesta en frecuencia tipo AUTOLAB PGSTAT30 en control potenciostático y al potencial libre de corrosión. Las medidas de impedancia fueron llevadas a cabo, en todos los casos, en un rango de frecuencias entre $1 \mathrm{mHz}$ y $100 \mathrm{kHz}$ con un voltaje sinusoidal de amplitud $10 \mathrm{mV}$ y en el interior de una celda de Faraday para evitar interferencias exteriores al sistema. Los espectros de impedancia se analizaron utilizando el software ZView. El circuito equivalente mostrado en la figura 1 se empleó para analizar el espectro de impedancias. 


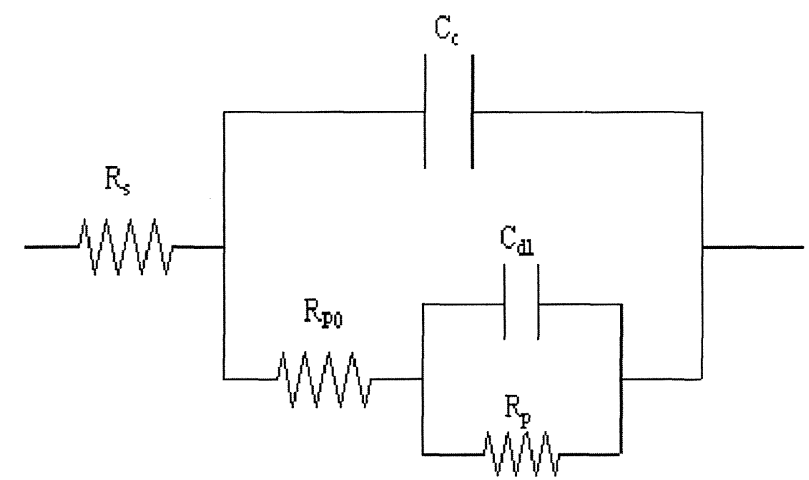

Figura 1. Circuito eléctrico equivalente.

Figure 1. Electric equivalent circuit.

\subsubsection{Ensayo acelerado (niebla salina)}

Se realizó según la norma ASTM B117-85 ${ }^{[4]}$, exponiendo las probetas a niebla salina durante diferentes periodos de tiempo, hasta $400 \mathrm{~h}$. Tras estas exposiciones, las probetas fueron evaluadas atendiendo a la deslaminación y al ampollamiento.

\subsubsection{Ensayo $A C / D C / A C$}

Este tipo de ensayo, como puede verse en la figura 2 , consiste en una combinación entre medidas de corriente continua (DC) y corriente alterna (AC). En primer lugar, se realiza un ensayo EIS para determinar el estado inicial de la muestra a analizar. Tras este ensayo, se aplica durante $30 \mathrm{~min}$ un potencial de corriente continua constante (DC) de $-3 \mathrm{~V}$ y a continuación se deja que la muestra relaje durante $3 \mathrm{~h}$, hasta alcanzar un nuevo estado de equilibrio. Por último se aplica un nuevo EIS para

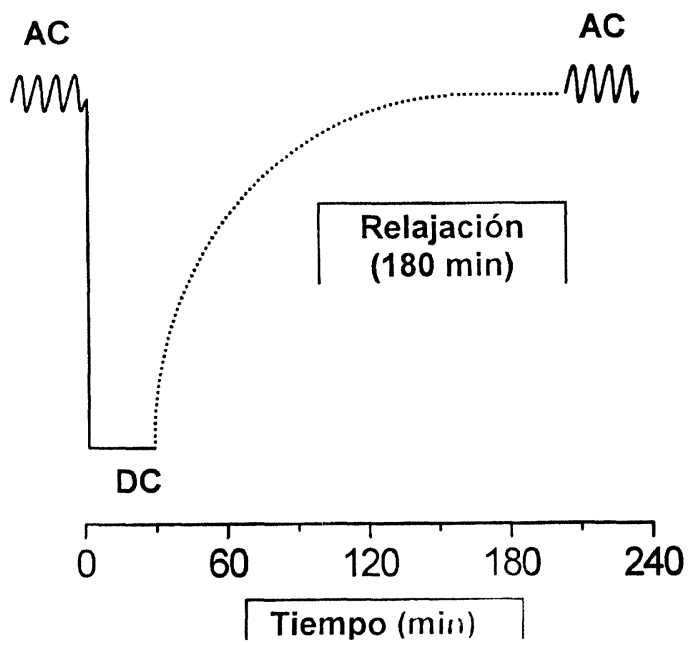

Figura 2. Ensayo $A C / D C / A C$.

Figure 2. $A C / D C / A C$ test. determinar el estado final, tras la polarización. Este ciclo, de aproximadamente $4 \mathrm{~h}$ de duración, se repitió durante $24 \mathrm{~h}$.

El ensayo AC/DC/AC se realizó en el equipo AUTOLAB PGSTAT30. Los espectros de impedancia obtenidos fueron modelizados mediante el software ZView utilizando el circuito equivalente de la figural.

\section{RESULTADOS}

\subsection{Análisis mecánico}

En la figura 3 se muestran las curvas tensión-deformación del ensayo de tracción para las diferentes imprimaciones. En la tabla II se indican los parámetros mecánicos obtenidos en estos ensayos. $\mathrm{Ob}$ servamos como el recubrimiento se vuelve más frágil y rígido y menos dúctil al aumentar CPV $\left(\mathrm{E}, \sigma_{\text {l.e. }}\right.$ y $\sigma_{\text {rot. }}$ aumentan, mientras que $\varepsilon_{\text {rot. }}$ disminuye), siendo esta tendencia especialmente significativa cuando CPV > CCPV.

\subsection{Ensayos electroquímicos}

Las figuras 4 y 5 representan los gráficos de Bode para los ensayos EIS y AC/DC/AC. Podemos observar como los resultados obtenidos mediante el ensayo AC/DC/AC muestran gran similitud con los hallados mediante el ensayo EIS. Así se encuentra que los valores más altos de impedancia (y por lo tanto, las mejores propiedades anticorrosivas) se dan en la imprimación con $\Lambda=0,73$, mientras que los menores valores son para $\Lambda=1,24$. En ambas figuras, se observa como, a medida que

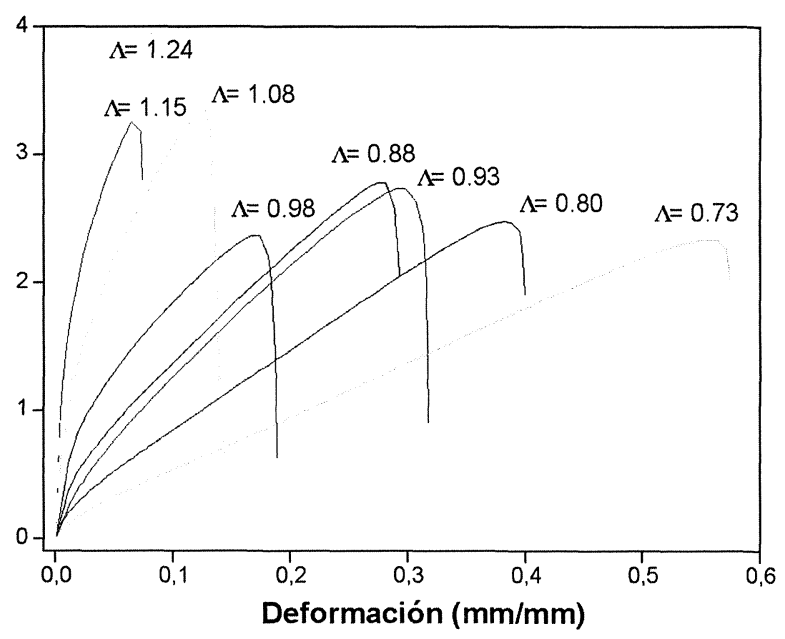

Figura 3. Curvas tensión-deformación para diferentes $\wedge$.

Figure 3. Stress-strain plot for different $\wedge$.

Rev. Metal. Madrid Vol. Extr. (2005) 202-207 
Tabla II. Parámetros mecánicos para diferentes $\Lambda$.

Table II. Mechanical parameters for different $\Lambda$.

\begin{tabular}{lrcccc}
\hline$\wedge$ & $\mathrm{E}(\mathrm{MPa})$ & $\sigma_{\text {l.e. }}(\mathrm{MPa})$ & $\varepsilon_{\text {l.e. }}(\%)$ & $\sigma_{\mathrm{r}}(\mathrm{MPa})$ & $\varepsilon_{\mathrm{r}}(\%)$ \\
\hline 0,73 & 9,532 & 0,163 & 0,017 & 2,240 & 0,552 \\
0,80 & 16,784 & 0,292 & 0,017 & 2,373 & 0,348 \\
0,88 & 26,790 & 0,488 & 0,019 & 2,869 & 0,299 \\
0,93 & 20,767 & 0,448 & 0,022 & 2,810 & 0,311 \\
0,98 & 46,141 & 0,610 & 0,017 & 2,452 & 0,168 \\
1,08 & 76,691 & 1,279 & 0,018 & 3,024 & 0,111 \\
1,15 & 248,067 & 1,798 & 0,010 & 3,568 & 0,063 \\
1,24 & 228,587 & 2,158 & 0,012 & 3,452 & 0,037 \\
\hline
\end{tabular}

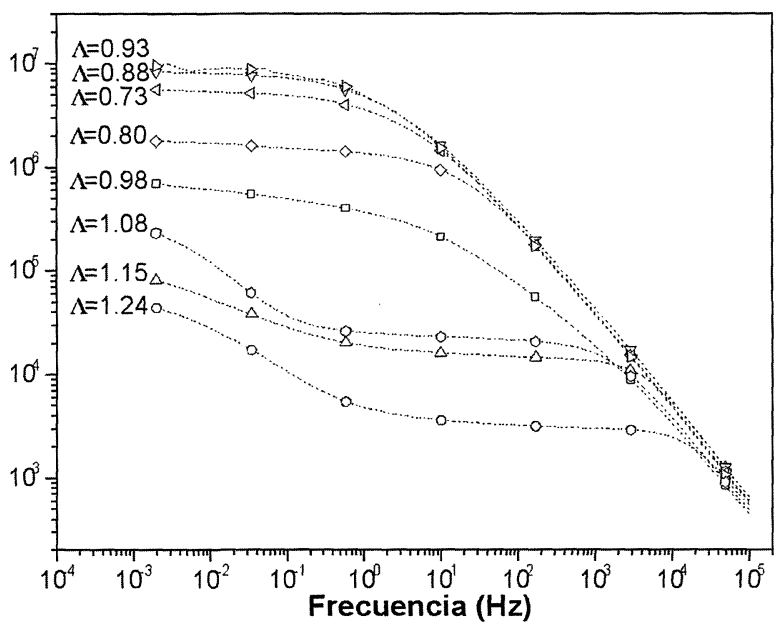

Figura 4. Diagrama de Bode, ensayo EIS, $14 \mathrm{~d}$.

Figure 4. Bode plot, EIS test, after $14 \mathrm{~d}$.

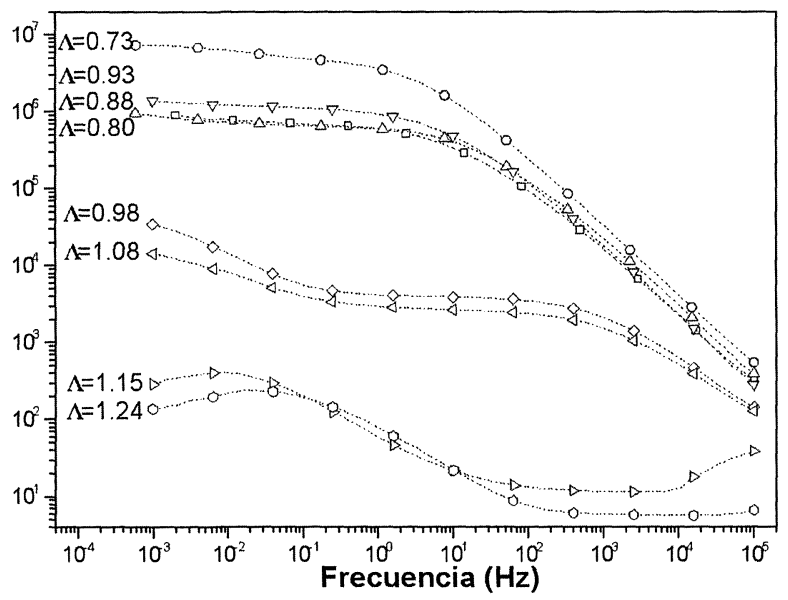

Figura 5. Diagrama de Bode, $A C / D C / A C, 5$ ciclos.

Figure 5. Bode plot, AC/DC/AC test, after 5 cycles.

aumenta el parámetro $\Lambda$, disminuye el módulo de la impedancia, siendo este descenso especialmente significativo para valores de $\Lambda>1$. Es importante destacar también que las imprimaciones con menor cantidad de pigmento presentan resultados de impedancia similares entre sí.

\subsection{Ensayos acelerados de niebla salina}

Tras exponer las muestras al ensayo de niebla salina durante diferentes periodos de tiempo, se procedió a medir la deslaminación que éstas habían sufrido (Fig. 6), obteniéndose menores deslaminaciones cuanto menor es CPV y aumentando ésta para valores de $\Lambda>1$.

\section{DISCUSIÓN}

\subsection{Propiedades mecánicas}

Un aumento de CPV en las imprimaciones trae consigo un fuerte incremento de rigidez en las muestras (tal y como se observa en la evolución del módulo de Young) y una pérdida significativa de elasticidad (disminución de la deformación a rotura). Estos cambios, son especialmente destacables cuando el valor del parámetro $\wedge$ es mayor que 1.

La explicación de la modificación de las propiedades mecánicas al aumentar CPV puede encontrarse en los efectos debidos a la inclusión de pigmentos. En primer lugar, los pigmentos pueden actuar como refuerzo en la matriz orgánica; por otro lado, pueden variar la densidad de entrecruzamiento de la resina epoxi; y, finalmente, pueden propiciar la entrada de aire en la matriz, ocasionando un cambio radical en las propiedades.

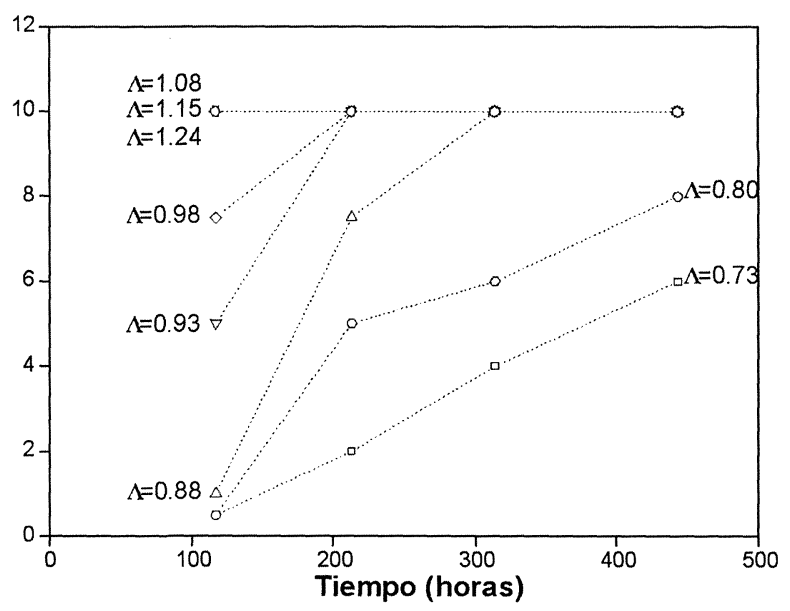

Figura 6. Ensayo de niebla salina para diferentes $\Lambda$.

Figure 6. Salt Fog Test for different $\Lambda$. 
En definitiva, un aumento de CPV origina una mayor rigidez y fragilidad en el recubrimiento orgánico y, dado que la imprimación estará sometida a tensiones significativas (proceso de curado, ciclos térmicos, absorción de agua...), se producirán procesos de deslaminación en la interfase metalrecubrimiento. Así pues, el aumento de CPV hará disminuir la adherencia de la imprimación al sustrato metálico.

\subsection{Propiedades anticorrosivas}

Las figuras 7 y 8 muestran la evolución del parámetro $\mathrm{C}_{\mathrm{dl}}$ para los ensayos electroquímicos EIS y AC/DC/AC. La capacitancia de doble capa $\mathrm{C}_{\mathrm{dl}}$, es

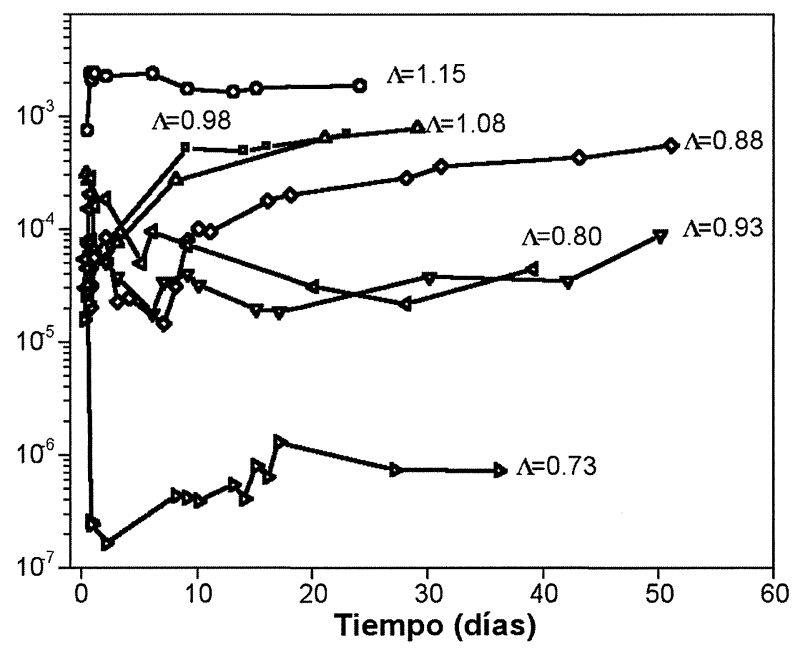

Figura 7. Evolución de la capacidad doble capa (EIS).

Figure 7. Time dependence of $C_{d l}$ (EIS).

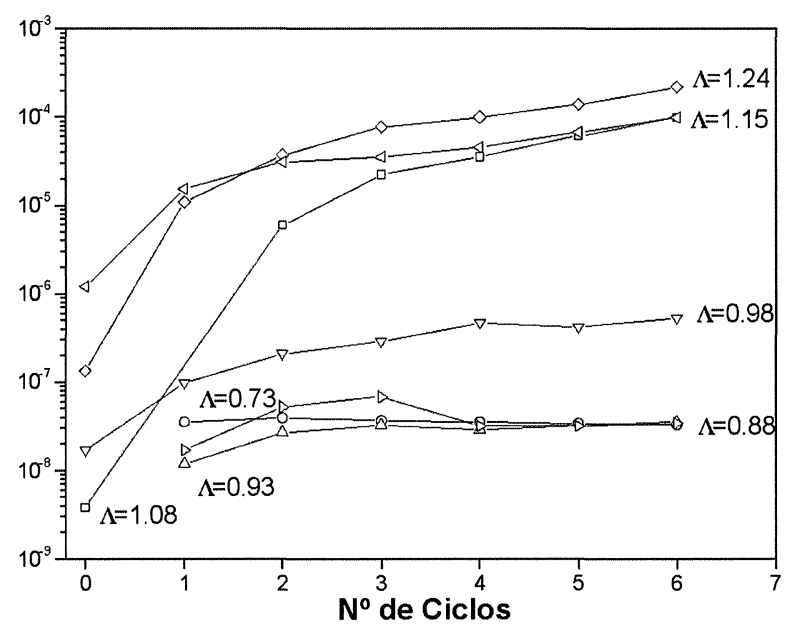

Figura 8. Evolución de la capacidad doble capa (AC/ $D C / A C)$.

Figure 8. Time dependence of $C_{d l}(A C / D C / A C$ test). una medida del área de deslaminación de la imprimación. Su variación es suma de dos efectos: $\mathrm{C}_{\mathrm{dl}}$ aumenta al llegar el electrolito a la interfase y disminuye por la acumulación de productos de corrosión que reducen el área de deslaminación. Debido a ello, una imprimación de buena calidad tendrá un valor constante de $C_{d}$, indicativo de una interfase estable y por lo tanto, poco activa, ya que cualquier variación de este parámetro pone de manifiesto la existencia de corrosión en la superficie metálica. Ambas técnicas electroquímicas sugieren que aumentos de CPV traen consigo un incremento de la deslaminación y, consecuentemente, una disminución de las propiedades anticorrosivas.

Estos resultados pueden ser explicados si tenemos en cuenta los dos tipos de protección anticorrosiva ofrecida por las imprimaciones: el efecto barrera, que impide la llegada del electrolito al sustrato metálico y la pasivación de la interfase. Un aumento de CPV implica una mayor adición de pigmento, lo que favorece la entrada de electrolito, hecho que no contrarresta el efecto inhibidor producido por la mayor presencia de pigmentos anticorrosivos.

En definitiva, los resultados indican que para este tipo de imprimación, las propiedades anticorrosivas tienden a mejorar conforme desciende el parámetro $\wedge$ (en nuestro caso hasta valores de $\Lambda=0,73)$.

Además, se observan cambios significativos en el comportamiento de las imprimaciones cuando CPV es mayor que CCPV, debido a que no existe suficiente resina para cubrir totalmente los pigmentos y las cargas, lo que genera un aumento de la permeabilidad por la formación de poros, produciendo una interfase mucho más activa. Los ensayos de niebla salina vienen a confirmar estos resultados, mostrando el aumento de la deslaminación conforme aumentamos la adición de pigmentos y especialmente a partir de valores de $\Lambda>1$.

\section{CONCLUSIONES}

En función de los resultados obtenidos se puede concluir que en las imprimaciones epoxis formuladas, las propiedades anticorrosivas y mecánicas se ven altamente afectadas por las variaciones del parámetro $\Lambda$, mejorando para valores bajos de $\Lambda$ y empeorando drásticamente al aumentar CPV por encima de $\Lambda>1$. Por lo tanto, en este tipo de imprimaciones interesa trabajar a bajos valores de CPV a pesar de la desventaja económica que esto supone, dado que al reducir el contenido en cargas se producirá un aumento del coste. 


\section{Agradecimientos}

Los autores agradecen a Eva Romero, Noelia Coll, Juan Carlos Galván, Ramón Novoa y David Roures su ayuda en el desarrollo experimental y al proyecto CICYT-MAT2000-0123-P4-03 su soporte económico.

\section{REFERENCIAS}

[1] W.K. Asbeck y M. VAN Loo, Ind. Eng. Chem. 41 (1949) 1470.

[2] R.W. Braunhausen, L. De Bolt y R.A. Baltrus, J. Coat. Technol. 64 (1992) 51-54.

[3] G.P. Bierwagen, J. Coat. Technol. 64 (1992) 71-75.

[4] Annual Book of ASTM Standards, Philadelphia, PA, (1985)

[5] B. Liu, Y. Li, H.C. Lin y C.N. CaO, Acta Phys-Chim. Sin. 17 (2001) 241-244.
[6] B.S. Skerry, C.T. ChEN y C.J. Ray, J. Coat. Technol. 64 (1992) 77-86.

[7] S. Gwori y K. Balakrishnan, Prog. Org. Coat. 23 (1994) 363-377.

[8] M. Selvaraj y S. Guruviah, Prog. Org. Coat. 28 (1996) 271-277.

[9] L.S. HeRnÁNDEZ y B. DEL Amo, Anti-Corros. Methods Mater. 46 (1999) 198-204.

[10] J. ShaO, P. Wan, X. Lin, H. Lin y S. ZhanG, Mater. Corros. 46 (1995) 33-38.

[11] J. Hollaender, E. Ludwig y S. Hillebrand, Proc. 5th Int. Tinplate Conf., London, (1992) 300.

[12] J. HOLLAENDER, Food additives and contaminants 14 (1997) 617.

[13] J. Hollaender, C.A. Schiller y W. Strunz, Proc. EIS 2001, Marilleva, Italy, 2001.

[14] C.H. Hare y J.S. Kurnas, J. Coat. Technol. 72 (2000) 21-27. 